Jahangirnagar University J. Biol. Sci. 7(1): 1-13, 2018 (June)

\title{
Wildlife Diversity of Madhupur National Park, Bangladesh
}

\author{
Monirujjaman* and M. Monirul H. Khan \\ Department of Zoology, Jahangirnagar University, Savar, Dhaka 1342, Bangladesh
}

\begin{abstract}
The study was conducted to know the wildlife diversity of Madhupur National Park of Bangladesh from June 2014 to March 2015. A total of 151 species of wildlife (amphibians to mammals) belonging to 23 orders and 62 families were recorded which covers $16.41 \%$ of total wildlife species in our country. Among them $10(7 \%)$ were amphibians, $15(10 \%)$ reptiles, $111(73 \%)$ birds and $15(10 \%)$ mammals while $65(43.05 \%)$ were very common, 41 (27.16\%) common, $25(16.55 \%)$ uncommon and $20(13.24 \%)$ were rare. Among the 20 species of migratory birds, $15(75 \%)$ were winter visitors and $5(25 \%)$ summer visitors. The forests and other wildlife habitats in and around Madhupur National Park are decreasing rapidly due to illegal logging, land conversion for agriculture and human settlements.
\end{abstract}

Key words: Diversity, wildlife, amphibian, reptile, bird, mammal, Madhupur National Park.

\section{INTRODUCTION}

In Bangladesh the diversity at the species level is commonly referred, because the species level can be sampled easily. This study was conducted to sample the wildlife diversity of Madhupur National Park in central Bangladesh and to compare the status across the different groups. Literally wildlife means the life forms living in the wild i.e., the life forms living independently of humans. Official definition of wildlife differs from country to country, but typically focuses on wild vertebrates except fish, i.e. mammals, birds, reptiles and amphibians. As many as 31 species of wildlife have been identified as Regionally Extinct (RE) by IUCN-Bangladesh (IUCN, 2015). Among them 11 species were mammals (Striped Hyena, Grey Wolf, Swamp Deer, Sloth Deer, Blackbuck, Nilgai, Banteng, Wild Water Buffalo, Sumatran Rhinoceros, Javan Rhinoceros, and Indian Rhinoceros), 19 birds (Rufous-throated Partridge, Indian Peafowl, Green Peafowl, Grey Francolin, Swamp Francolin, White-winged Duck, Pink-headed Duck, Sarus Crane, Bengal Florican, Lesser Florican, Greater Adjutant, White-bellied Heron, Spot-billed Pelican, Red-headed Vulture, Rusty-fronted Barwing, Black-breasted Parrotbill, Spotbreasted Parrotbill, Rufous-headeded Parrotbill, and Bar-tailed Treecreeper), and 1 reptile (Marsh Crocodile). Some relevant works have been done on the birds of Madhupur forest or Tangail district (Islam 1983, Khan \& Islam 2000, Haque 1975, Khan \& Ahsan 2011.

Study Area: Madhupur National Park is the largest deciduous forest of Bangladesh, located in the Madhupur Garh, comprises an area of 8436 ha (DoE, 2015 \& IUCN, 2015). The Park is situated in the northeastern part of Tangail Forest Division along the

\footnotetext{
*Corresponding author. E-mail: monirujjamankhan912@gmail.com
} 
boundary with Mymensingh district (it also extends slightly into the district) and $125 \mathrm{~km}$ away from Dhaka. It is located from $24^{\circ} 36^{\prime}$ to $24^{\circ} 42^{\prime}$ North latitudes and $90^{\circ} 00^{\prime}$ to $90^{\circ} 06^{\prime}$ East longitudes (Figure 1). The altitude of the park is about $20 \mathrm{~m}$ above the mean sea level (Khan \& Ahsan, 2011). Madhupur National Park began as Madhupur Shal (Shorea robusta) Forest, but was finally declared as National Park on 24 February 1982 (Bangladesh Forest Department 2015), although justify for national park status began as early as 1962 (Bangladesh Forest Department 2015).

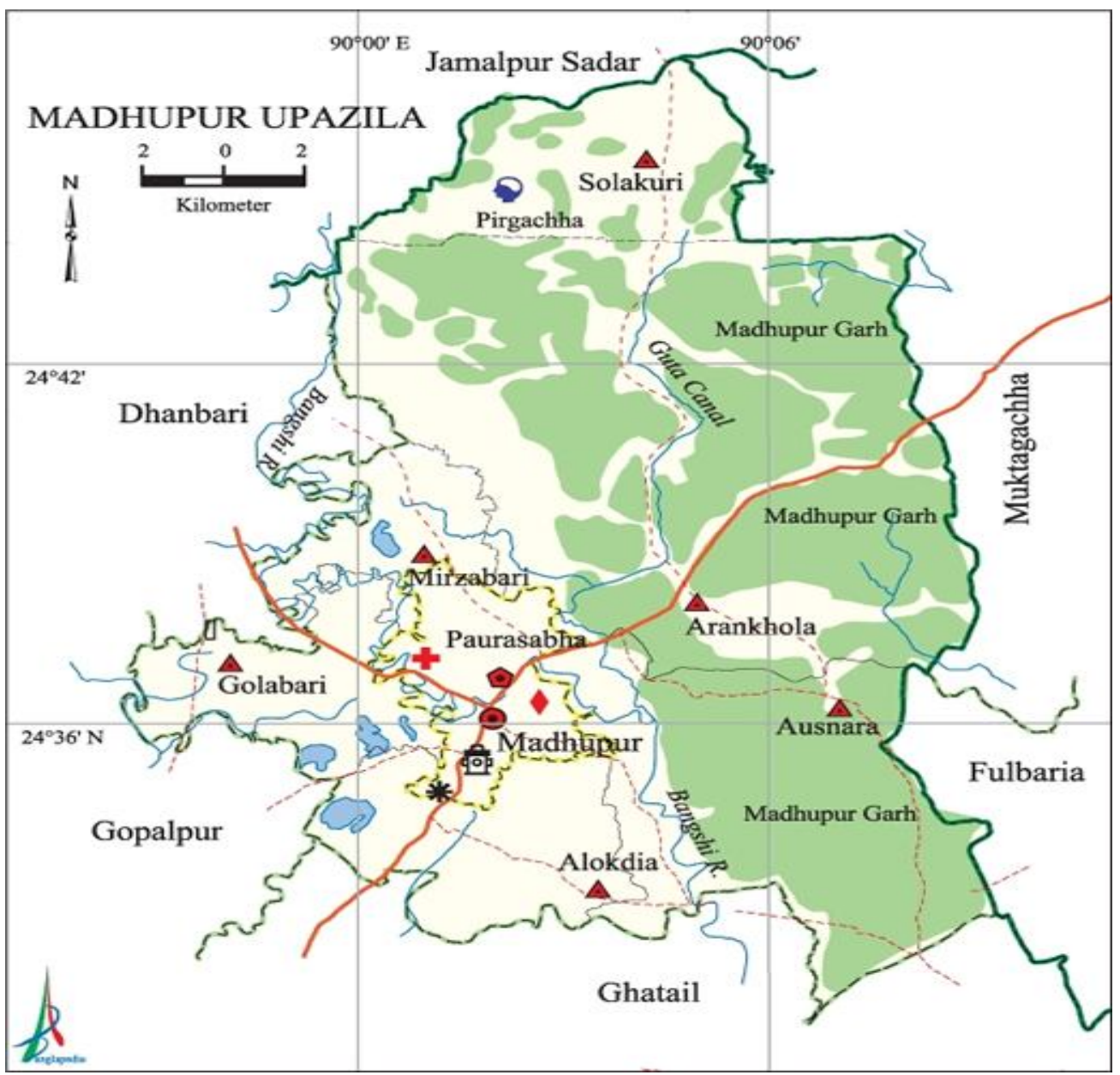

Fig. 1. Map of Madhupur Upazila showing the Madhupur National Park

\section{MATERIALS AND METHODS}

Study on wildlife diversity of Madhupur National Park was done from June 2014 to March 2015. The observation was made for four or five days per month. A total of 47 days (spending 510 hours) were spent to collect data. The observations started early in the 
morning, immediate after sunrise and continued after sunset till $8.00 \mathrm{pm}$, with midday break. Wildlife species were identified by using Encyclopedia of Flora and Fauna Bangladesh, 2009, references books and publications (Grimmett 2011, Halder 2010, Hasan 2014, Khan 2008). The methods that were followed are plot counting, line transect sampling and interviews of local people.

Direct Observation: Materials and Methods used by the authors, Daniel (1963), Husain \& Rahman (1978), Rahman (1985), Sarker \& Sarker (1985), Khan (1998) were used in the direct field observation -

Line-transect Sampling: Data regarding the different species available in the study areas were recorded by line-transect sampling. Counting was conducted along the roads in the villages and cultivated lands.

Plot Counting: To observe the amphibian fauna, plot counting methods were used.

Calls and Songs: Some bird species which were normally not hut seen, but recorded by hearing songs and calls.

The observation and data collection methods used for different groups of wildlife are described below -

Amphibians: Plot counting method was applied for collecting data about amphibian species. Each of $300 \mathrm{~m} \times 300 \mathrm{~m}$ of plots were selected in the study area and they were further divided into few mini transect lines.

Reptiles: For the lizards and snakes plot counting method and line-transect method as well as information through questionnaire were used. The questionnaire based information could only provide the presence and abundance of the species.

Birds: Only line-transect method was applied for collecting data on birds. The length and width of each transect line were $500 \mathrm{~m}$ and $30 \mathrm{~m}$, respectively.

Mammals: For the information about the mammalian species line-transect method, plot counting method and information through questionnaire were used. Plot counting method was preferably applied for counting rats, mice, monkeys, shrews, and so on.

Four categories were used to express the status of different species (Khan, 2008): Very Common (VC) - a species seen during 76-100\% of the visits; Common (C) - a species seen during $51-75 \%$ of the visits; Uncommon (UC) - a species seen during $26-50 \%$ of the visits; and Rare (R) - a species seen single or in small number of occasions, i.e. up to $25 \%$ of the visits when it is most active.

\section{RESULTS AND DISCUSSION}

Overall 151 species of wildlife (amphibians to mammals) belonging to 23 orders and 62 families were recorded in the Madhupur National Park (Table 1-4), which is (16.41\%) of 
the total wildlife 920 species (IUCN, 2015) in Bangladesh. Among these $10(7 \%)$ were amphibians, $15(10 \%)$ reptiles, $111(73 \%)$ birds, and $15(10 \%)$ mammals. Out of 151 species, $65(43.05 \%)$ were very common, $25(16.55 \%)$ uncommon, $20(13.24 \%)$ rare and $41(27.16 \%)$ common (Figure 2$)$. Birds were relatively very common than others and are easily noticeable than other species of wildlife.

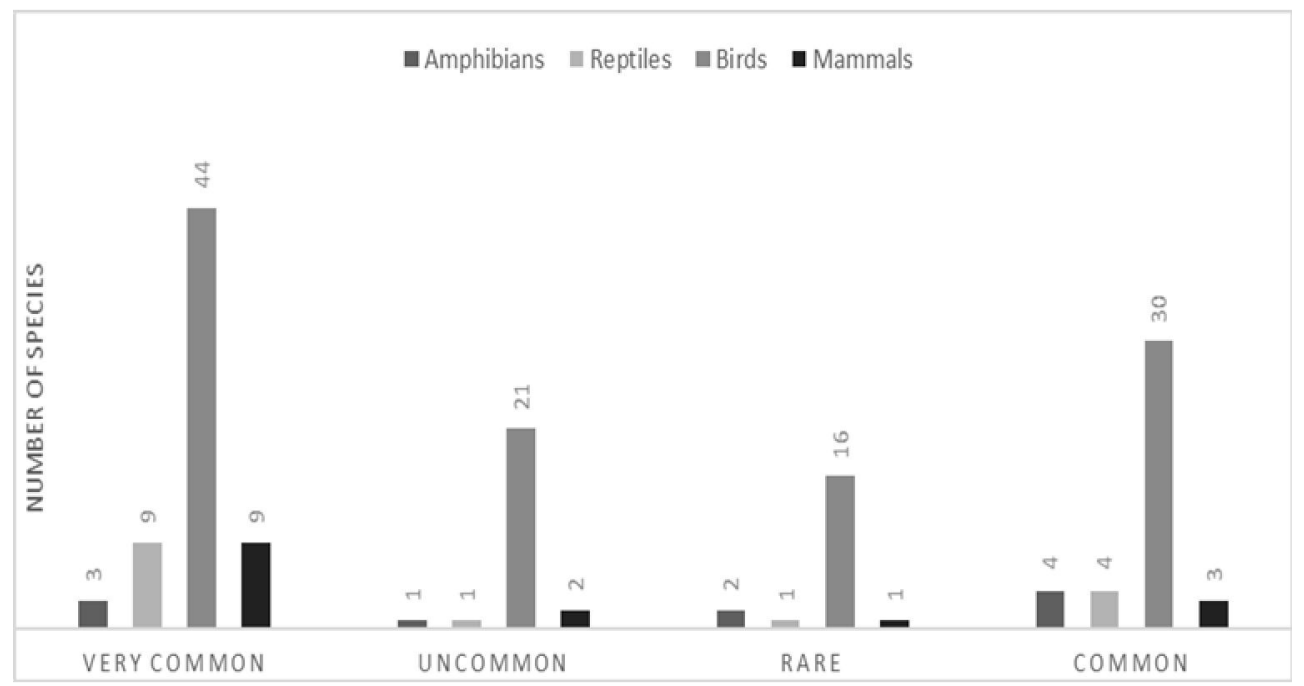

Fig. 2. Number of very common, uncommon, rare, and common species of amphibians, reptiles birds and mammals

Ninety six species of wildlife were recorded in the starting month (June 2014) of this study and 151 species at the end of (March 2015). The monthly cumulative frequency of total number of wildlife species (Figure 3) indicates near to saturation, though might be changed by long-term study.

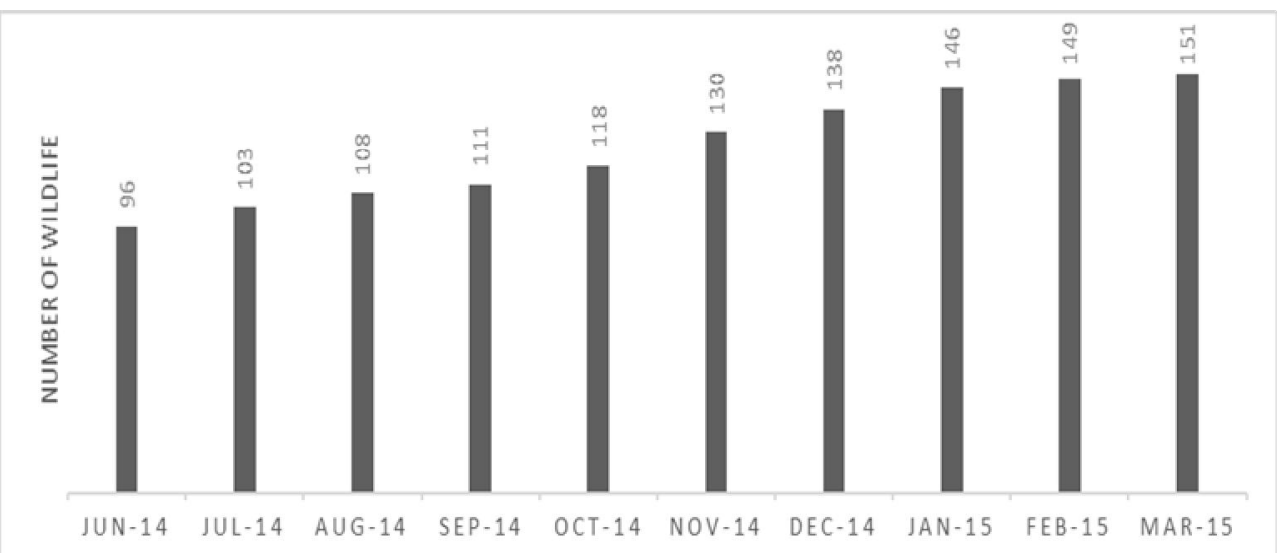

Fig. 3. Total number of wildlife species (cumulative) recorded in Madhupur National Park showing the saturation of recorded 
Amphibians: Ten species of anuran amphibians under 5 families (Bufonidae, Dicroglossidae, Ranidae, Microhylidae and Rhacophoridae) were recorded (Table 1). The Madhupur National Park supports (20.4\%) amphibians considering 49 species from Bangladesh (IUCN, 2015). Of the recorded species $1(10 \%)$ was toad and $9(90 \%)$ were frogs; among them 3 (30\%) were very common, 4 (40\%) common, 1 (10\%) uncommon, 2 $(20 \%)$ rare species.

Table 1. Amphibians observed in the Madhupur National Park during June 2014 to March 2015

\begin{tabular}{|c|c|c|c|c|}
\hline $\begin{array}{l}\text { Sl } \\
\text { No. }\end{array}$ & Common name & Scientific name & Family & Status \\
\hline \multicolumn{5}{|c|}{ Order: Anura } \\
\hline 01 & Common Asian Toad & $\begin{array}{l}\text { Duttaphrynus melanostictus } \\
\text { (Schneider, 1799) }\end{array}$ & Bufonida & $\mathrm{VC}$ \\
\hline 02 & Skipper Frog & $\begin{array}{l}\text { Euphlyctis cyanophlyctis } \\
\text { (Schneider, 1799) }\end{array}$ & Dicroglossidae & $\mathrm{VC}$ \\
\hline 03 & Green Pond Frog & $\begin{array}{l}\text { Euphlyctis hexadactylus (Lesson, } \\
1834 \text { ) }\end{array}$ & Dicroglossidae & $\mathrm{R}$ \\
\hline 04 & Terai Cricket Frog & $\begin{array}{l}\text { Fejervarya teraiensis (Dubois, } \\
\text { 1984) }\end{array}$ & Dicroglossidae & $\mathrm{C}$ \\
\hline 05 & Jerdon's Bull Frog & $\begin{array}{l}\text { Hoplobatrachus crassus (Jerdon, } \\
1853 \text { ) }\end{array}$ & Dicroglossidae & $\mathrm{R}$ \\
\hline 06 & Indian Bull Frog & $\begin{array}{l}\text { Hoplobatrachus tigerinus (Daudin, } \\
\text { 1803) }\end{array}$ & Dicroglossidae & $\mathrm{VC}$ \\
\hline 07 & Cope's Frog & Hylarana leptoglossa (Cope, 1868) & Ranidae & $\mathrm{C}$ \\
\hline 08 & $\begin{array}{l}\text { Mymensingh } \\
\text { Microhylid Frog }\end{array}$ & $\begin{array}{l}\text { Microhyla mymensinghensis } \\
\text { (Hasan, 2014) }\end{array}$ & Microhylidae & $\mathrm{C}$ \\
\hline 09 & Indian Balloon Frog & $\begin{array}{l}\text { Uperodon globulosus (Gunther, } \\
1864 \text { ) }\end{array}$ & Microhylidae & $\mathrm{C}$ \\
\hline 10 & Indian Tree Frog & $\begin{array}{l}\text { Polypedates maculatus (J.E.Gray, } \\
\text { 1830) }\end{array}$ & Rhacophoridae & $\mathrm{UC}$ \\
\hline
\end{tabular}

Reptiles: Fifteen species of reptiles under 2 orders (Squamata and Serpentes) and 7 families (Agamidae, Varanidae, Elapidae, Typhlopidae, Scinidae, Colubridae, and Gekkonidae) were recorded (Table 2). The Madhupur National Park supports (8.98\%) reptiles considering 168 species from Bangladesh (IUCN, 2015). Of the recorded species $1(7 \%)$ was monitor, $5(33 \%)$ were lizards, $3(20 \%)$ skinks and $6(40 \%)$ snakes; among them 9 (60\%) were very common, 4 (26.66\%) common, 1 (6.67\%) uncommon, $1(6.67 \%)$ rare species. Turtle is rare in the study area, no turtle was observed during the study period. Many reptiles, particularly snakes and lizards, play an important role in biological pest control by consumily insects and rodents that are harmful for crops. 
Table 2. Reptiles observed in the Madhupur National Park during June 2014 to March 2015

\begin{tabular}{|c|c|c|c|c|}
\hline $\begin{array}{l}\text { Sl } \\
\text { No. }\end{array}$ & Common name & Scientific name & Family & Status \\
\hline \multicolumn{5}{|c|}{ Order: Squamata } \\
\hline 01 & $\begin{array}{l}\text { Common Garden } \\
\text { Lizard }\end{array}$ & Calotes versicolor (Daudin, 1802) & Agamidae & $\mathrm{VC}$ \\
\hline 02 & Tokay Gecko & Gekko gecko (Linnaeus, 1758) & Gekkonidae & $\mathrm{C}$ \\
\hline 03 & $\begin{array}{l}\text { Common House } \\
\text { Gecko }\end{array}$ & $\begin{array}{l}\text { Hemidactylus frenatus (Schlegel, } \\
\text { 1836) }\end{array}$ & Gekkonidae & $\mathrm{VC}$ \\
\hline 04 & $\begin{array}{l}\text { Flat-tailed House } \\
\text { Gecko }\end{array}$ & $\begin{array}{l}\text { Hemidactylus platyurus } \\
\text { (Schneider, 1792) }\end{array}$ & Gekkonidae & $\mathrm{UC}$ \\
\hline 05 & Brook's House Gecko & Hemidactylus brookii (Gray, 1845) & Gekkonidae & $\mathrm{R}$ \\
\hline 06 & Keeled Grass Skink & $\begin{array}{l}\text { Mabuya carinata (Schneider, } \\
1801 \text { ) }\end{array}$ & Scincidae & $\mathrm{VC}$ \\
\hline 07 & Bronze Grass Skink & Mabuya macularia (Blyth, 1853) & Scincidae & $\mathrm{C}$ \\
\hline 08 & $\begin{array}{l}\text { Bowring's Supple } \\
\text { Skink }\end{array}$ & $\begin{array}{l}\text { Lygosoma bowringii } \\
\text { (Cogger,1994) }\end{array}$ & Scincidae & $\mathrm{C}$ \\
\hline 09 & Bengal Monitor & $\begin{array}{l}\text { Varanus bengalensis (Daudin, } \\
1802 \text { ) }\end{array}$ & Varanidae & $\mathrm{VC}$ \\
\hline \multicolumn{5}{|c|}{ Order: Serpentes } \\
\hline 10 & Diard's Blind Snake & Typhlops diardii (Schlegel, 1839) & Typhlopidae & $\mathrm{VC}$ \\
\hline 11 & Jerdon's Blind Snake & $\begin{array}{l}\text { Typhlops jerdoni (Boulenger, } \\
\text { 1890) }\end{array}$ & Typhlopidae & $\mathrm{C}$ \\
\hline 12 & Common Vine Snake & Ahaetulla nasuta (Lacépède, 1789) & Colubridae & $\mathrm{VC}$ \\
\hline 13 & Checkered Keelback & $\begin{array}{l}\text { Xenochrophis piscator (Schneider, } \\
1799 \text { ) }\end{array}$ & Colubridae & $\mathrm{VC}$ \\
\hline 14 & $\begin{array}{l}\text { Common Smooth } \\
\text { Water Snake }\end{array}$ & $\begin{array}{l}\text { Enhydris enhydris (Schneider, } \\
\text { 1799) }\end{array}$ & Colubridae & $\mathrm{VC}$ \\
\hline 15 & Spectacled Cobra & Naja naja (Linnaeus, 1758) & Elapidae & $\mathrm{VC}$ \\
\hline
\end{tabular}

Birds: One hundred and eleven species of birds under 13 orders (Galliformes, Anseriformes, Piciformes, Upupiformes, Coraciformes, Cuculiformes, Psittaciformes, Apodiformes, Strigiformes, Columbiformes, Gruiformes, Ciconiiformes, and Passeriformes) and 38 families were recorded (Table 3). Among these 59 (53.15\%) were non-passerines where $52(46.85 \%)$ were passerines. The Madhupur National Park supports (19.61\%) birds considering 566 species from Bangladesh (IUCN, 2015). Of the recorded species $94(84.69 \%)$ were resident and $17(15.31 \%)$ migratory birds. Among migratory birds, $13(76.47 \%)$ were winter visitors and 4 (23.53\%) summer visitors. The number of very common birds were $44(39.64 \%)$, whereas 30 (27.03\%) common, 21 $(18.91 \%)$ uncommon, and $16(14.42 \%)$ rare species. 
Table 3. Birds observed in the Madhupur National Park during June 2014 to March 2015

\begin{tabular}{|c|c|c|c|c|}
\hline $\begin{array}{l}\text { Sl. } \\
\text { No }\end{array}$ & Common name & Scientific name & Family & Status \\
\hline \multicolumn{5}{|c|}{ Order: Galliformes } \\
\hline 01 & Red Junglefowl & Gallus gallus (Linnaeus, 1758) & Phasianidae & $\mathrm{C}, \mathrm{L}$ \\
\hline \multicolumn{5}{|c|}{ Order: Anseriformes } \\
\hline 02 & $\begin{array}{l}\text { Fulvous Whistling- } \\
\text { duck }\end{array}$ & $\begin{array}{l}\text { Dendrocygna bicolor (Vieillot, } \\
\text { 1816) }\end{array}$ & Dendrocygnidae & $\mathrm{R}, \mathrm{L}$ \\
\hline 03 & Northern Pintail & Anas acuta (Linnaeus, 1758) & Anatidae & $\mathrm{R}, \mathrm{Mw}$ \\
\hline 04 & Spot-billed Duck & $\begin{array}{l}\text { Anas poecilorhyncha (Forster, } \\
\text { 1781) }\end{array}$ & Anatidae & $\mathrm{R}, \mathrm{L}$ \\
\hline 05 & Cotton Pygmy-goose & $\begin{array}{l}\text { Nettapus coromandelianus } \\
\text { (Gmelin, 1789) }\end{array}$ & Anatidae & $\mathrm{R}, \mathrm{L}$ \\
\hline \multicolumn{5}{|c|}{ Order: Piciformes } \\
\hline 06 & Rufous Woodpecker & $\begin{array}{l}\text { Celeus brachyurus (Vieillot, } \\
1818 \text { ) }\end{array}$ & Picidae & VC, L \\
\hline 07 & Greater Flameback & $\begin{array}{l}\text { Chrysocolaptes guttacristatus } \\
\text { (Tickell, 1833) }\end{array}$ & Picidae & $\mathrm{C}, \mathrm{L}$ \\
\hline 08 & $\begin{array}{l}\text { Grey-capped Pygmy } \\
\text { Woodpecker }\end{array}$ & $\begin{array}{l}\text { Dendrocopos canicapillus } \\
\text { (Blyth, 1845) }\end{array}$ & Picidae & $\mathrm{VC}, \mathrm{L}$ \\
\hline 09 & $\begin{array}{l}\text { Fulvous-breasted } \\
\text { Woodpecker }\end{array}$ & $\begin{array}{l}\text { Dendrocopos macei (Vieillot, } \\
\text { 1818) }\end{array}$ & Picidae & $\mathrm{VC}, \mathrm{L}$ \\
\hline 10 & $\begin{array}{l}\text { Black-rumped } \\
\text { Flamback }\end{array}$ & $\begin{array}{l}\text { Dinopium benghalense } \\
\text { (Linnaeus, 1758) }\end{array}$ & Picidae & $\mathrm{C}, \mathrm{L}$ \\
\hline 11 & $\begin{array}{l}\text { Streak-throated } \\
\text { Woodpecker }\end{array}$ & $\begin{array}{l}\text { Picus xanthopygaeus (Gray \& } \\
\text { Gray, 1847) }\end{array}$ & Picidae & $\mathrm{R}, \mathrm{L}$ \\
\hline 12 & Blue-throated Barbet & $\begin{array}{l}\text { Psilopogon asiaticus (Latham, } \\
\text { 1790) }\end{array}$ & Megalaimidae & $\mathrm{VC}, \mathrm{L}$ \\
\hline 13 & Coppersmith Barbet & $\begin{array}{l}\text { Psilopogon haemacephalus } \\
\text { (Statius Muller, 1776) }\end{array}$ & Megalaimidae & VC, L \\
\hline \multicolumn{5}{|c|}{ Order: Upupiformes } \\
\hline 14 & Common Hoopoe & Upupa epops (Linnaeus, 1758) & Upupidae & $\mathrm{C}, \mathrm{L}$ \\
\hline \multicolumn{5}{|c|}{ Order: Coraciformes } \\
\hline 15 & Indian Roller & $\begin{array}{l}\text { Coracias benghalensis } \\
\text { (Linnaeus, 1758) }\end{array}$ & Coraciidae & $\mathrm{VC}, \mathrm{L}$ \\
\hline 16 & Common Kingfisher & Alcedo atthis (Linnaeus, 1758) & Alcedinidae & $\mathrm{VC}, \mathrm{L}$ \\
\hline 17 & $\begin{array}{l}\text { White-throated } \\
\text { Kingfisher }\end{array}$ & $\begin{array}{l}\text { Halcyon smyrnensis (Linnaeus, } \\
\text { 1758) }\end{array}$ & Halcyonidae & $\mathrm{C}, \mathrm{L}$ \\
\hline 18 & $\begin{array}{l}\text { Stork-billed } \\
\text { Kingfisher }\end{array}$ & $\begin{array}{l}\text { Pelargopsis capensis (Linnaeus, } \\
\text { 1758) }\end{array}$ & Halcyonidae & $\mathrm{C}, \mathrm{L}$ \\
\hline 19 & $\begin{array}{l}\text { Chestnut-headed } \\
\text { Bee-eater }\end{array}$ & $\begin{array}{l}\text { Merops leschenaulti (Vieillot, } \\
\text { 1817) }\end{array}$ & Meropidae & $\mathrm{C}, \mathrm{L}$ \\
\hline 20 & Green Bee-eater & $\begin{array}{l}\text { Merops orientalis (Latham, } \\
\text { 1801) }\end{array}$ & Meropidae & $\mathrm{C}, \mathrm{L}$ \\
\hline 21 & Blue-tailed Bee-eater & $\begin{array}{l}\text { Merops philippinus (Linnaeus, } \\
1766)\end{array}$ & Meropidae & $\mathrm{C}, \mathrm{Ms}$ \\
\hline \multicolumn{5}{|c|}{ Order: Cuculiformes } \\
\hline 22 & Jacobin Cuckoo & Clamator jacobinus (Boddaert, & Cuculidae & $\mathrm{C}, \mathrm{Ms}$ \\
\hline
\end{tabular}




\begin{tabular}{|c|c|c|c|c|}
\hline \multicolumn{5}{|c|}{$\begin{array}{r}1783) \\
\end{array}$} \\
\hline 23 & Indian Cuckoo & $\begin{array}{l}\text { Cuculus micropterus (Gould, } \\
\text { 1837) }\end{array}$ & Cuculidae & $\mathrm{C}, \mathrm{Ms}$ \\
\hline 24 & Asian Koel & $\begin{array}{l}\text { Endynamys scolopaceus } \\
\text { (Linnaeus, 1758) }\end{array}$ & Cuculidae & $\mathrm{VC}, \mathrm{L}$ \\
\hline 25 & Lesser Coucal & $\begin{array}{l}\text { Centropus bengalensis (Gmelin, } \\
\text { 1788) }\end{array}$ & Centropodidae & $\mathrm{C}, \mathrm{L}$ \\
\hline 26 & Greater Coucal & $\begin{array}{l}\text { Centropus sinensis (Stephens, } \\
\text { 1815) }\end{array}$ & Centropodidae & $\mathrm{C}, \mathrm{L}$ \\
\hline \multicolumn{5}{|c|}{ Order: Psittaciformes } \\
\hline 27 & $\begin{array}{l}\text { Red-breasted } \\
\text { Parakeet }\end{array}$ & $\begin{array}{l}\text { Psittacula alexandri (Linnaeus, } \\
\text { 1758) }\end{array}$ & Psittacidae & $\mathrm{VC}, \mathrm{L}$ \\
\hline 28 & Rose-ringed Parakeet & $\begin{array}{l}\text { Psittacula krameri (Scopoli, } \\
\text { 1769) }\end{array}$ & Psittacidae & $\mathrm{VC}, \mathrm{L}$ \\
\hline \multicolumn{5}{|c|}{ Order: Apodiformes } \\
\hline 29 & Asian Palm Swift & $\begin{array}{l}\text { Cypsiurus balasiensis (J.E. } \\
\text { Gray, 1829) }\end{array}$ & Apodidae & $\mathrm{C}, \mathrm{L}$ \\
\hline \multicolumn{5}{|c|}{ Order: Strigiformes } \\
\hline 30 & Barn Owl & Tyto alba (Scopoli, 1769) & Tytonidae & VC, L \\
\hline 31 & Spotted Owlet & $\begin{array}{l}\text { Athene brama } \text { (Temminck, } \\
\text { 1821) }\end{array}$ & Strigidae & $\mathrm{R}, \mathrm{Mw}$ \\
\hline 32 & Dusky Eagle Owl & $\begin{array}{l}\text { Bubo coromandus (Latham, } \\
1790 \text { ) }\end{array}$ & Strigidae & $\mathrm{R}, \mathrm{L}$ \\
\hline 33 & Brown Fish Owl & $\begin{array}{l}\text { Ketupa zeylonensis (Gmelin, } \\
1788 \text { ) }\end{array}$ & Strigidae & $\mathrm{UC}, \mathrm{L}$ \\
\hline 34 & Brown Hawk Owl & Ninox scutulata (Raffles, 1822) & Strigidae & $\mathrm{UC}, \mathrm{L}$ \\
\hline \multicolumn{5}{|c|}{ Order: Columbiformes } \\
\hline 35 & Emerald Dove & $\begin{array}{l}\text { Chalcophaps indica (Linnaeus, } \\
\text { 1758) }\end{array}$ & Columbidae & $\mathrm{UC}, \mathrm{L}$ \\
\hline 36 & Rock Pigeon & Columba livia (Gmelin, 1789) & Columbidae & VC, L \\
\hline 37 & Spotted Dove & $\begin{array}{l}\text { Streptopelia chinensis (Scopoli, } \\
1768 \text { ) }\end{array}$ & Columbidae & VC, L \\
\hline 38 & $\begin{array}{l}\text { Eurasian Collared } \\
\text { Dove }\end{array}$ & $\begin{array}{l}\text { Streptopelia decaocto } \\
\text { (Frivaldszky, 1838) }\end{array}$ & Columbidae & $\mathrm{UC}, \mathrm{L}$ \\
\hline 39 & Red Collared Dove & $\begin{array}{l}\text { Streptopelia tranquebarica } \\
\text { (Hermann, 1804) }\end{array}$ & Columbidae & $\mathrm{UC}, \mathrm{L}$ \\
\hline 40 & $\begin{array}{l}\text { Orange-breasted } \\
\text { Green Pigeon }\end{array}$ & Treron bicincta (Jerdon, 1840) & Columbidae & $\mathrm{R}, \mathrm{L}$ \\
\hline 41 & $\begin{array}{l}\text { Yellow-footed Green } \\
\text { Pigeon }\end{array}$ & $\begin{array}{l}\text { Treron phoenicoptera (Latham, } \\
\text { 1790) }\end{array}$ & Columbidae & $\mathrm{VC}, \mathrm{L}$ \\
\hline \multicolumn{5}{|c|}{ Order: Gruiformes } \\
\hline 42 & $\begin{array}{l}\text { White-breasted } \\
\text { Waterhen }\end{array}$ & $\begin{array}{l}\text { Amaurornis phoenicurus } \\
\text { (Pennant, 1769) }\end{array}$ & Rallidae & $\mathrm{UC}, \mathrm{L}$ \\
\hline 43 & Common Coot & Fulica atra (Linnaeus, 1758) & Rallidae & $\mathrm{UC}, \mathrm{L}$ \\
\hline 44 & Purple Swamphen & $\begin{array}{l}\text { Porphyrio porphyrio (Linnaeus, } \\
1758 \text { ) }\end{array}$ & Rallidae & $\mathrm{R}, \mathrm{Mw}$ \\
\hline \multicolumn{5}{|c|}{ Order: Ciconiiformes } \\
\hline 45 & Bronze-winged & Metopidius indicus (Latham, & Jacanidae & $\mathrm{UC}, \mathrm{L}$ \\
\hline
\end{tabular}




\begin{tabular}{|c|c|c|c|c|}
\hline & Jacana & 1790) & & \\
\hline 46 & Red-wattled Lapwing & $\begin{array}{l}\text { Vanellus indicus (Boddaert, } \\
1783 \text { ) }\end{array}$ & Charadriidae & $\mathrm{UC}, \mathrm{L}$ \\
\hline 47 & Brahminy Kite & $\begin{array}{l}\text { Haliastur indus (Boddaert, } \\
1783 \text { ) }\end{array}$ & Accipitridae & $\mathrm{VC}, \mathrm{L}$ \\
\hline 48 & Black Kite & $\begin{array}{l}\text { Milvus migrans (Boddaert, } \\
1783 \text { ) }\end{array}$ & Accipitridae & $\mathrm{VC}, \mathrm{L}$ \\
\hline 49 & Great Cormorant & $\begin{array}{l}\text { Phalacrocorax carbo (Linnaeus, } \\
1758 \text { ) }\end{array}$ & Phalacrocoracidae & $\mathrm{C}, \mathrm{L}$ \\
\hline 50 & Little Cormorant & $\begin{array}{l}\text { Phalacrocorax niger (Vieillot, } \\
\text { 1817) }\end{array}$ & Phalacrocoracidae & $\mathrm{C}, \mathrm{L}$ \\
\hline 51 & Gray Heron & Ardea cinerea (Linnaeus, 1758) & Ardeidae & $\mathrm{C}, \mathrm{L}$ \\
\hline 52 & Indian Pond Heron & Ardeola grayii (Sykes, 1832) & Ardeidae & VC, $\mathrm{L}$ \\
\hline 53 & Cattle Egret & Bubulcus ibis (Linnaeus, 1758) & Ardeidae & VC, L \\
\hline 54 & Little Heron & $\begin{array}{l}\text { Butorides striatus (Linnaeus, } \\
1758 \text { ) }\end{array}$ & Ardeidae & $\mathrm{VC}, \mathrm{L}$ \\
\hline 55 & Great Egret & Ardea alba (Linnaeus, 1758) & Ardeidae & VC, L \\
\hline 56 & Little Egret & $\begin{array}{l}\text { Egretta garzetta (Linnaeus, } \\
1766 \text { ) }\end{array}$ & Ardeidae & $\mathrm{VC}, \mathrm{L}$ \\
\hline 57 & $\begin{array}{l}\text { Black-crowned Night } \\
\text { Heron }\end{array}$ & $\begin{array}{l}\text { Nycticorax nycticorax } \\
\text { (Linnaeus, 1758) }\end{array}$ & Ardeidae & $\mathrm{UC}, \mathrm{L}$ \\
\hline 58 & Asian Openbill & $\begin{array}{l}\text { Anastomus oscitans (Boddaert, } \\
\text { 1783) }\end{array}$ & Ciconiidae & $\mathrm{C}, \mathrm{L}$ \\
\hline 59 & Lesser Adjutant & $\begin{array}{l}\text { Leptoptilos javanicus (Horsfield, } \\
\text { 1821) }\end{array}$ & Ciconiidae & $\mathrm{R}, \mathrm{L}$ \\
\hline \multicolumn{5}{|c|}{ Order: Passeriformes } \\
\hline 60 & Indian Pitta & $\begin{array}{l}\text { Pitta brachyura (Linnaeus, } \\
\text { 1758) }\end{array}$ & Pittidae & $\mathrm{C}, \mathrm{Ms}$ \\
\hline 61 & $\begin{array}{l}\text { Golden-fronted } \\
\text { Leafbird }\end{array}$ & $\begin{array}{l}\text { Chloropsis aurifrons } \\
\text { (Temminck, 1829) }\end{array}$ & Irenidae & $\mathrm{UC}, \mathrm{L}$ \\
\hline 62 & Brown Shrike & $\begin{array}{l}\text { Lanius cristatus (Linnaeus, } \\
1758 \text { ) }\end{array}$ & Laniidae & $\begin{array}{l}\mathrm{UC}, \\
\mathrm{Mw}\end{array}$ \\
\hline 63 & Long-tailed Shrike & Lanius schach (Linnaeus, 1758) & Laniidae & $\mathrm{VC}, \mathrm{L}$ \\
\hline 64 & Gray-backed Shrike & $\begin{array}{l}\text { Lanius tephronotus (Vigors, } \\
1831 \text { ) }\end{array}$ & Laniidae & $\begin{array}{l}\mathrm{VC} \\
\mathrm{Mw}\end{array}$ \\
\hline 65 & Common Iora & $\begin{array}{l}\text { Aegithina tiphia (Linnaeus, } \\
1758 \text { ) }\end{array}$ & Corvidae & VC, L \\
\hline 66 & Large-billed Crow & $\begin{array}{l}\text { Corvus macrorhynchos (Wagler, } \\
\text { 1827) }\end{array}$ & Corvidae & $\mathrm{VC}, \mathrm{L}$ \\
\hline 67 & House Crow & $\begin{array}{l}\text { Corvus splendens (Vieillot, } \\
\text { 1817) }\end{array}$ & Corvidae & $\mathrm{VC}, \mathrm{L}$ \\
\hline 68 & Rufous Treepie & $\begin{array}{l}\text { Dendrocitta vagabunda } \\
\text { (Latham, 1790) }\end{array}$ & Corvidae & VC, L \\
\hline 69 & Bronzed Drongo & Dicrurus aeneus (Vieillot, 1817) & Corvidae & VC, $\mathrm{L}$ \\
\hline 70 & Spangled Drongo & $\begin{array}{l}\text { Dicrurus bracteatus (Gould, } \\
1842 \text { ) }\end{array}$ & Corvidae & UC, $\mathrm{L}$ \\
\hline 71 & Ashy Drongo & $\begin{array}{l}\text { Dicrurus leucophaeus (Vieillot, } \\
\text { 1817) }\end{array}$ & Corvidae & $\mathrm{C}, \mathrm{Mw}$ \\
\hline 72 & Black Drongo & $\begin{array}{l}\text { Dicrurus macrocercus (Vieillot, } \\
1817 \text { ) }\end{array}$ & Corvidae & $\mathrm{VC}, \mathrm{L}$ \\
\hline
\end{tabular}




\begin{tabular}{|c|c|c|c|c|}
\hline 73 & $\begin{array}{l}\text { Greater Racket-tailed } \\
\text { Drongo }\end{array}$ & $\begin{array}{l}\text { Dicrurus paradiseus (Linnaeus, } \\
1766 \text { ) }\end{array}$ & Corvidae & $\mathrm{R}, \mathrm{L}$ \\
\hline 74 & $\begin{array}{l}\text { Bar-winged } \\
\text { Flycatcher-shrike }\end{array}$ & Hemipus picatus (Sykes, 1832) & Corvidae & $\mathrm{C}, \mathrm{L}$ \\
\hline 75 & Black-naped Oriole & $\begin{array}{l}\text { Oriolus chinensis (Linnaeus, } \\
1766 \text { ) }\end{array}$ & Corvidae & $\mathrm{C}, \mathrm{Mw}$ \\
\hline 76 & Black-hooded Oriole & $\begin{array}{l}\text { Oriolus xanthornus (Linnaeus, } \\
1758 \text { ) }\end{array}$ & Corvidae & $\mathrm{VC}, \mathrm{L}$ \\
\hline 77 & Small Minivet & $\begin{array}{l}\text { Pericrocotus cinnamomeus } \\
\text { (Linnaeus, 1766) }\end{array}$ & Corvidae & $\mathrm{C}, \mathrm{L}$ \\
\hline 78 & White-rumped Shama & $\begin{array}{l}\text { Copsychus malabaricus } \\
\text { (Scopoli, 1788) }\end{array}$ & Muscicapidae & $\mathrm{VC}, \mathrm{L}$ \\
\hline 79 & $\begin{array}{l}\text { Oriental Magpie } \\
\text { Robin }\end{array}$ & $\begin{array}{l}\text { Copsychus saularis (Linnaeus, } \\
1758 \text { ) }\end{array}$ & Muscicapidae & $\mathrm{VC}, \mathrm{L}$ \\
\hline 80 & $\begin{array}{l}\text { Gray-headed Canary } \\
\text { Flycatcher }\end{array}$ & $\begin{array}{l}\text { Culicicapa ceylonensis } \\
\text { (Swainson, 1820) }\end{array}$ & Muscicapidae & $\begin{array}{l}\mathrm{UC}, \\
\mathrm{Mw}\end{array}$ \\
\hline 81 & Common Stonechat & $\begin{array}{l}\text { Saxicola torquatus (Linnaeus, } \\
1766 \text { ) }\end{array}$ & Muscicapidae & $\begin{array}{l}\mathrm{UC} \\
\mathrm{Mw}\end{array}$ \\
\hline 82 & $\begin{array}{l}\text { Orange-headed } \\
\text { Thrush }\end{array}$ & $\begin{array}{l}\text { Geokichla citrine (Latham, } \\
\text { 1790) }\end{array}$ & Muscicapidae & $\mathrm{C}, \mathrm{L}$ \\
\hline 83 & Jungle Myna & $\begin{array}{l}\text { Acridotheres fuscus (Wagler, } \\
\text { 1872) }\end{array}$ & Sturnidae & $\mathrm{VC}, \mathrm{L}$ \\
\hline 84 & Bank Myna & $\begin{array}{l}\text { Acridotheres ginginianus } \\
\text { (Latham, 1790) }\end{array}$ & Sturnidae & $\mathrm{VC}, \mathrm{L}$ \\
\hline 85 & Common Myna & $\begin{array}{l}\text { Acridotheres tristis (Linnaeus, } \\
\text { 1766) }\end{array}$ & Sturnidae & $\mathrm{C}, \mathrm{L}$ \\
\hline 86 & Asian Pied Starling & $\begin{array}{l}\text { Gracupica contra (Linnaeus, } \\
1758 \text { ) }\end{array}$ & Sturnidae & $\mathrm{VC}, \mathrm{L}$ \\
\hline 87 & $\begin{array}{l}\text { Chestnut-tailed } \\
\text { Starling }\end{array}$ & $\begin{array}{l}\text { Sturnus malabarica (Gmelin, } \\
\text { 1789) }\end{array}$ & Sturnidae & $\mathrm{VC}, \mathrm{L}$ \\
\hline 88 & Great Tit & Parus major (Linnaeus, 1758) & Sittidae & $\mathrm{UC}, \mathrm{L}$ \\
\hline 89 & Plain Martin & $\begin{array}{l}\text { Riparia paludicola (Vieillot, } \\
\text { 1817) }\end{array}$ & Hirundinidae & $\mathrm{UC}, \mathrm{L}$ \\
\hline 90 & Red-vented Bulbul & $\begin{array}{l}\text { Pycnonotus cafer (Linnaeus, } \\
1766 \text { ) }\end{array}$ & Pycnonotidae & $\mathrm{VC}, \mathrm{L}$ \\
\hline 91 & $\begin{array}{l}\text { Red-whiskered } \\
\text { Bulbul }\end{array}$ & $\begin{array}{l}\text { Pycnonotus jocosus (Linnaeus, } \\
1758 \text { ) }\end{array}$ & Pycnonotidae & $\mathrm{C}, \mathrm{L}$ \\
\hline 92 & Zitting Cisticola & $\begin{array}{l}\text { Cisticola juncidis (Rafinesque, } \\
1810 \text { ) }\end{array}$ & Cisticolidae & $\mathrm{C}, \mathrm{L}$ \\
\hline 93 & Common Tailorbird & $\begin{array}{l}\text { Orthotomus sutorius (Pennant, } \\
\text { 1769) }\end{array}$ & Sylviidae & $\mathrm{VC}, \mathrm{L}$ \\
\hline 94 & Jungle Babbler & $\begin{array}{l}\text { Turdoides striata (Dumont, } \\
1823 \text { ) }\end{array}$ & Sylviidae & $\mathrm{VC}, \mathrm{L}$ \\
\hline 95 & Crimson Sunbird & $\begin{array}{l}\text { Aethopyga siparaja (Raffles, } \\
\text { 1822) }\end{array}$ & Nectariniidae & $\mathrm{C}, \mathrm{L}$ \\
\hline 96 & $\begin{array}{l}\text { Ruby-cheeked } \\
\text { Sunbird }\end{array}$ & $\begin{array}{l}\text { Chalcoparia singalensis } \\
\text { (Gmelin, 1788) }\end{array}$ & Nectariniidae & $\mathrm{UC}, \mathrm{L}$ \\
\hline 97 & Purple Sunbird & $\begin{array}{l}\text { Cinnyris asiaticus (Latham, } \\
\text { 1790) }\end{array}$ & Nectariniidae & $\mathrm{VC}, \mathrm{L}$ \\
\hline
\end{tabular}




\begin{tabular}{|c|c|c|c|c|}
\hline 98 & $\begin{array}{l}\text { Purple-rumped } \\
\text { Sunbird }\end{array}$ & $\begin{array}{l}\text { Leptocoma zeylonica (Linnaeus, } \\
1766)\end{array}$ & Nectariniidae & $\mathrm{C}, \mathrm{L}$ \\
\hline 99 & Siberian Rubythroat & Calliope calliope (Pallas, 1776) & Turdidae & $\mathrm{UC}, \mathrm{L}$ \\
\hline 100 & Tawny Pipit & $\begin{array}{l}\text { Anthus campstris (Linnaeus, } \\
\text { 1758) }\end{array}$ & Passeridae & $\mathrm{R}, \mathrm{Mw}$ \\
\hline 101 & Olive-backed Pipit & $\begin{array}{l}\text { Anthus hodgsoni (Richmond, } \\
\text { 1907) }\end{array}$ & Passeridae & $\begin{array}{l}\mathrm{UC}, \\
\mathrm{Mw}\end{array}$ \\
\hline 102 & Paddyfield Pipit & Anthus rufulus (Vieillot, 1818) & Passeridae & $\mathrm{VC}, \mathrm{L}$ \\
\hline 103 & Indian Silverbill & $\begin{array}{l}\text { Euodice malabarica (Linnaeus, } \\
1758 \text { ) }\end{array}$ & Passeridae & $\mathrm{R}, \mathrm{L}$ \\
\hline 104 & Black-headed Munia & $\begin{array}{l}\text { Lonchura atricapilla (Vieillot, } \\
\text { 1807) }\end{array}$ & Passeridae & $\mathrm{C}, \mathrm{L}$ \\
\hline 105 & Scaly-breasted Munia & $\begin{array}{l}\text { Lonchura punctulata (Linnaeus, } \\
\text { 1758) }\end{array}$ & Passeridae & $\mathrm{VC}, \mathrm{L}$ \\
\hline 106 & White Wagtail & Motacilla alba (Linnaeus, 1758) & Passeridae & $\mathrm{R}, \mathrm{Mw}$ \\
\hline 107 & Gray Wagtail & $\begin{array}{l}\text { Motacilla cinerea (Tunstall, } \\
\text { 1771) }\end{array}$ & Passeridae & $\mathrm{R}, \mathrm{Mw}$ \\
\hline 108 & $\begin{array}{l}\text { White-browed } \\
\text { Wagtail }\end{array}$ & $\begin{array}{l}\text { Motacilla maderaspatensis } \\
\text { (Gmelin, 1789) }\end{array}$ & Passeridae & $\mathrm{UC}, \mathrm{L}$ \\
\hline 109 & House Sparrow & $\begin{array}{l}\text { Passer domesticus (Linnaeus, } \\
1758 \text { ) }\end{array}$ & Passeridae & $\mathrm{VC}, \mathrm{L}$ \\
\hline 110 & $\begin{array}{l}\text { Eurasian Tree } \\
\text { Sparrow }\end{array}$ & $\begin{array}{l}\text { Passer montanus (Linnaeus, } \\
1758 \text { ) }\end{array}$ & Passeridae & $\mathrm{R}, \mathrm{L}$ \\
\hline 111 & Baya Weaver & $\begin{array}{l}\text { Ploceus philippinus (Linnaeus, } \\
\text { 1766) }\end{array}$ & Passeridae & VC, L \\
\hline
\end{tabular}

Mammals: Fifteen species of mammals under 7 orders, and 12 families were recorded (Table 4). The Madhupur National Park supports (10.87\%) mammals considering 138 species from Bangladesh (IUCN, 2015). Among these 9 (60\%) were very common, 3 (20\%) common, $2(13.33 \%)$ uncommon, $1(6.67 \%)$ rare species. Barking Deer was uncommon in natural habitat, but very common in captive deer breeding centre in Lahoria, which is situated in Madhupur National Park. Eurasian Wild Boar was common in this area a few years ago, but no record was found in recent years. The reason might be illegal hunting by Garo community.

Table 4. Mammals observed in the Madhupur National Park during June 2014 to March 2015

\begin{tabular}{l|l|l|l|l}
\hline $\begin{array}{l}\text { Sl } \\
\text { No }\end{array}$ & Common name & Scientific name & Family & Status \\
\hline \multicolumn{2}{l}{ Order: Lagomorpha } & & \\
\hline 01 & Indian Hare & Lepus nigricollis (F. Cuvier, 1823) & Leporidae & UC \\
\hline Order: Rodentia & & \\
\hline 02 & Irrawaddy Squirrel & $\begin{array}{l}\text { Callosciurus pygerythrus (I. } \\
\text { Geoffroy Saint Hilaire, 1832) }\end{array}$ & Sciuridae \\
03 & $\begin{array}{l}\text { Asiatic Long Tailed } \\
\text { Climbing Mouse }\end{array}$ & $\begin{array}{l}\text { Vandeleuria oleracea (Bennett, } \\
\text { 1832) }\end{array}$ & Muridae & VC \\
04 & Greater Bandicoot- & Bandicota indica (Bechstein, & Muridae & VC \\
\hline
\end{tabular}




\begin{tabular}{|c|c|c|c|c|}
\hline \multirow{2}{*}{\multicolumn{2}{|c|}{$\frac{\text { rat }}{\text { Order: Carnivora }}$}} & \multicolumn{3}{|l|}{ 1800) } \\
\hline & & & & \\
\hline 05 & Large Indian Civet & Viverra zibetha (Linnaeus, 1758) & Viverridae & $\mathrm{C}$ \\
\hline 06 & Jangal Cat & Felis chaus (Schreber, 1777) & Felidae & VC \\
\hline 07 & Fishing Cat & $\begin{array}{l}\text { Prionailurus viverrinus (Bennett, } \\
\text { 1833) }\end{array}$ & Felidae & $\mathrm{C}$ \\
\hline 08 & $\begin{array}{l}\text { Small Indian } \\
\text { Mongoose }\end{array}$ & $\begin{array}{l}\text { Herpestes auropunctatus } \\
\text { (Hodgson, 1836) }\end{array}$ & Herpestidae & $\mathrm{VC}$ \\
\hline 09 & Golden Jackal & Canis aureus (Linnaeus, 1758) & Canidae & VC \\
\hline \multicolumn{5}{|c|}{ Order: Chiroptera } \\
\hline 10 & Indian Flying Fox & $\begin{array}{l}\text { Pteropus giganteus (Brünnich, } \\
\text { 1782) }\end{array}$ & Pteropodidae & $\mathrm{VC}$ \\
\hline 11 & Indian Pipistrelle & $\begin{array}{l}\text { Pipistrellus coromandra (Gray, } \\
1838 \text { ) }\end{array}$ & Vespertilionidae & $\mathrm{C}$ \\
\hline \multicolumn{5}{|c|}{ Order: Primates } \\
\hline 12 & Rhesus Macaque & $\begin{array}{l}\text { Macaca mulatta (Zimmermann, } \\
\text { 1780) }\end{array}$ & Cercopithecidae & $\mathrm{VC}$ \\
\hline 13 & Capped Langur & $\begin{array}{l}\text { Trachypithecus pileatus (Blyth, } \\
1843 \text { ) }\end{array}$ & Cercopithecidae & VC \\
\hline \multicolumn{5}{|c|}{ Order: Scandentia } \\
\hline 14 & $\begin{array}{l}\text { Common Tree } \\
\text { Shrew }\end{array}$ & $\begin{array}{l}\text { Tupaia glis (Diard \& Duvaucel, } \\
\text { 1820) }\end{array}$ & Tupaiidae & $\mathrm{R}$ \\
\hline \multicolumn{5}{|c|}{ Order: Artiodactyla } \\
\hline 15 & Barking Deer & $\begin{array}{l}\text { Muntiacus muntjak (Zimmermann, } \\
\text { 1780) }\end{array}$ & Cervidae & $\mathrm{UC}$ \\
\hline
\end{tabular}

The study area covered (14.36\%) of total wildlife species. Husain and Haque (1977) reported the occurrence of 170 species (and 1 subspecies) from the Madhupur forest in Tangail and Mymensingh districts. Khan \& Ahsan (2011) reported that, 115 species of birds were found in Madhupur National Park, under the 12 orders, 36 families and 87 genera. Khan (1998), studied the taxonomy and ecology of the birds of Tangail and recorded a total of 216 species of birds under 48 families has been registered which represents nearly one-third of the total bird species found in Bangladesh. Out of 216 species, 59 (27\%) were migratory and 157 (73\%) resident. Husain (1991) reported the occurrence of 169 species of birds from the Jamuna Multipurpose Bridge Area of Tangail and Sirajganj. There is no significant difference between the number of non-passerines, passerines, resident and migratory birds recorded in 2011 and the present study year. Although the previous research by Hssain \& Haque (1977), Husain (1991) and Khan (1998) reveals that wildlife populations here decreased remarkably at present.

Once Eurasian Wild Boar, Tiger, Leopard, Sambar Deer, Wild Water Buffalo, Red Jungle Fowl, Peacock, Turtle, Green Frog and many other animals were very common, but now because of continuous hunting by the Garo community, these species are becoming rare in this forest (Zaman, 2015). Habitat loss, illegal hunting, trapping and collecting of wild species, random use of agrochemicals, and lack of awareness were identified as threats to wildlife species in Madhupur National Park. Human encroachment, illegal hunting or 
trapping of wild animal should be controlled by creating public awareness to conserve wildlife of Madhupur National Park.

\section{REFERENCES}

Ahmed, Z.U., Begum, Z.N.T., Hassan, M.A., Khondker, M., Rahman, M.M., Kabir, S.M.H., Ahmad, M., Ahmed, A.T.A., Rahman, A.K.A. and Hoque, E.U. 2007-2009. Encyclopedia of Flora and Fauna of Bangladesh. Vols 1-28. Asiatic Society of Bangladesh. Dhaka.

Bangladesh Forest Department 2015. Bangladesh Wildlife Conservation Master Plan 2015-2035. Bangladesh Forest Department, Dhaka, Bangladesh.

Daniel, J.C. 1963. A Field Guide of the Amphibians of Western India Part-1. Journal of Bombay Natural Historical Society. 60(2): 415-438.

Department of Environment. 2015. Fifth National Report to the Convention on Biological Diversity. Department of Environment, Ministry of Environment and Forests, Government of the People's Republic Bangladesh, Dhaka. pp. 162.

Grimmett, R., Inskipp, C. and Inskipp, T. 2011. Birds of Indian Subcontinent. Christopher Helm, London, UK.

Halder, R.R. 2010. A Photographic Guide to Birds of Bangladesh. Baikal Teal Production, Dhaka, Bangladesh.

Haque, M.N. 1975. The avifauna of Madhupur Forest (With notes on ecology, status, distribution and food). Diss. M. Sc. thesis (unpublished), Dhaka University, Dhaka.

Hasan, M.K., Khan, M.M.H. and Feeroz, M.M. 2014. Amphibians and Reptiles of BangladeshA field Guide. Arannayk Foundation, Dhaka, Bangladesh.

Husain, K.Z. and Haque, M.N. 1977. The avifauna of the Madhupur forest. Proceedings First Bangladesh National Conference on Forestry, Dhaka. pp. 381-392.

Husain, K.Z. and Rhaman, M.M. 1978. The Amphibian Fauna of Bangladesh. Bangladesh Journal of Zoology. 6(2): 157-158.

Husain, K.Z., Rahman, M.K. and Haque, M.N. 1991. Wildlife diversity of the Jamuna Multipurpose Bridge area (a folder). Wildlife Society of Bangladesh, C/O. Department of Zoology, University of Dhaka, Dhaka.

IUCN- Bangladesh. 2015. Red List of Bangladesh. Vol. 1: Summary. IUCN- Bangladesh, Dhaka, Bangladesh.

Islam, M.A. 1983. On some birds of Tangail district (with notes on taxonomy and ecology). M.Sc. Thesis (unpublished). Dhaka University, Dhaka.

Khan, M.M.H. 1998. Taxonomy and Ecology of Birds of Tangail. M.Sc. Dissertation (Unpublished), Jahangirnagar University, Savar.

Khan, M.M.H. 2008. Protected Areas of Bangladesh- A Guide to Wildlife. Nishorgo Program, Bangladesh Forest Department. pp. 10-29.

Khan, M.M.H. 2014. Introduction to the Wildlife of Bangladesh. Jahangirnagar University and Bangladesh Forest Department. pp. 5-29.

Khan, M.M.H. and Islam, M.A. 2000. Status and habitats of the birds of Tangail, Bangladesh. Bangladesh J. Zool. 28(1): 75-88.

Khan, S.I. and Ahsan, M.F. 2011. Birds of the Madhupur National Park, Bangladesh. Bangladesh J. Zool. 39(1): 49-63.

Sarker, S.U and Sarker, N.J. 1985. Wildlife of Bangladesh, A Systematic list. The Rico Pricters.

Zaman, M. 2015. Wildlife diversity and indigenous knowledge of the Garo community in and around Madhupur National Park of Bangladesh. M.Sc. Dissertation (Unpublished), Jahangirnagar University, Savar. pp. 15-66. 